\title{
Isolated Pulmonary Metastasis Metastasectomy After Curative Prostate Cancer Treatment in Oligometastatic Disease
}

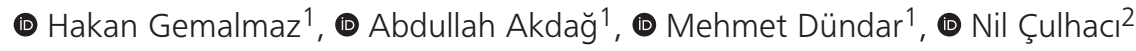 \\ ${ }^{1}$ Adnan Menderes University Hospital, Clinic of Urology, Aydın, Turkey \\ ${ }^{2}$ Adnan Menderes University Hospital, Clinic of Pathology, Aydın, Turkey
}

\begin{abstract}
Isolated pulmonary metastasis is observed in $2 \%-3 \%$ of prostate cancer cases but a complete treatment algorithm was not established for these patients. This study aimed to present a case of isolated pulmonary metastasis during the follow-up after radical prostatectomy, in which recurrence was not detected for 2 years after metastasectomy. The patient was on follow-up without any treatment for 22 months, with an unobservable prostate-specific antigen value. Metastasectomy in oligometastatic disease has emerged as a treatment option in recent years but is not considered a standard treatment. Literature contribution is necessary for oligometastatic disease definition to clarify its nature and compare treatment options.
\end{abstract}

Keywords: Prostate cancer, metastasis, recurrence, metastasectomy

\section{Introduction}

Isolated lung metastasis is observed in 2\%-3\% of prostate cancer (PCa) cases, without an established complete treatment algorithm for these patients (1). The lymph nodes and bones are the most common metastatic sites of $\mathrm{PCa}$; however, visceral metastasis rates are not negligible. In addition, visceral involvement represents a more aggressive disease (2). A recent prospective study revealed beneficial imaging-guided metastasis-based therapies in patients with recurrent PCa after primary treatment (3). Most metastases have nodal and bone involvement, thus salvage therapies are directed. The role of resection in pulmonary metastases is still unclear. This study aimed to present a case of isolated pulmonary metastasis during the follow-up after radical prostatectomy, in which recurrence was not detected for 2 years after metastasectomy.

\section{Case Report}

A 60-year-old male patient presented with penile deviation and pain. Rectal examination revealed a $5 \mathrm{~mm}$ rigid nodule in the right lobe of the prostate. Prostate-specific antigen (PSA) and free PSA were $2.6 \mathrm{ng} / \mathrm{mL}$ and $0.35 \mathrm{ng} / \mathrm{mL}$, respectively. Systematic ten quadrant biopsies with transrectal ultrasonography were performed, and Gleason 3+3=6 (15\%$30 \%$ ) prostate adenocarcinoma was diagnosed in two samples. In November 2010, a radical prostatectomy was performed. The final pathology revealed a Gleason 3+4=7 prostate adenocarcinoma located in the posterior right lobe and anterior left lobe. The lesion was located in $20 \%$ of the prostate with the largest size of $1.5 \mathrm{~cm}$. The tumor reached the capsule, with lymphovascular and perineural invasion in the tumoral areas and high-grade prostatic intraepithelial neoplasia in the adjacent areas. The tumor continued in the anterior surgical margin area. Immunohistochemical high molecular weight keratin staining of the anterior surgical margin had no staining. Posterior surgical margin, ductus deferens, and seminal vesicle were intact. The pathological TNM stage was reported as pt2c.

The first-month postoperative PSA was $0.03 \mathrm{ng} / \mathrm{mL}$. Upon PSA detection of $0.052 \mathrm{ng} / \mathrm{mL}$ in November 2012, 0.089 $\mathrm{ng} / \mathrm{mL}$ in March 2013, and $0.18 \mathrm{ng} / \mathrm{mL}$ in June 2013, the patient underwent abdominal computerized tomography and total body bone scintigraphy for metastasis screening. Metastasis signs were not found. Between June and August 2013, 72cGy salvage radiotherapy was given. Thereafter, PSA 
decreased to $0.003 \mathrm{ng} / \mathrm{mL}$. When the PSA value was $0.4 \mathrm{ng} /$ $\mathrm{mL}$ in February 2017, metastasis screening was performed with F-18 fluorodeoxyglucose positron emission tomography. A hypermetabolic $2 \mathrm{~cm}$ nodule was detected in the middle lobe of the right lung (Figure 1). The patient was evaluated for second, primary, or metastasis, thus segmental lobectomy was decided. In October 2017, right lung middle lobectomy and lymph node dissection pathology were reported as adenocarcinoma metastasis, without lymph node involvement (Figure 2). The patient was on follow-up for 22 months without any treatment. The PSA value is unobservable $(0.003 \mathrm{ng} / \mathrm{mL}$, July 31,2019$)$. The patient's information was presented as a case report after obtaining patient consent.

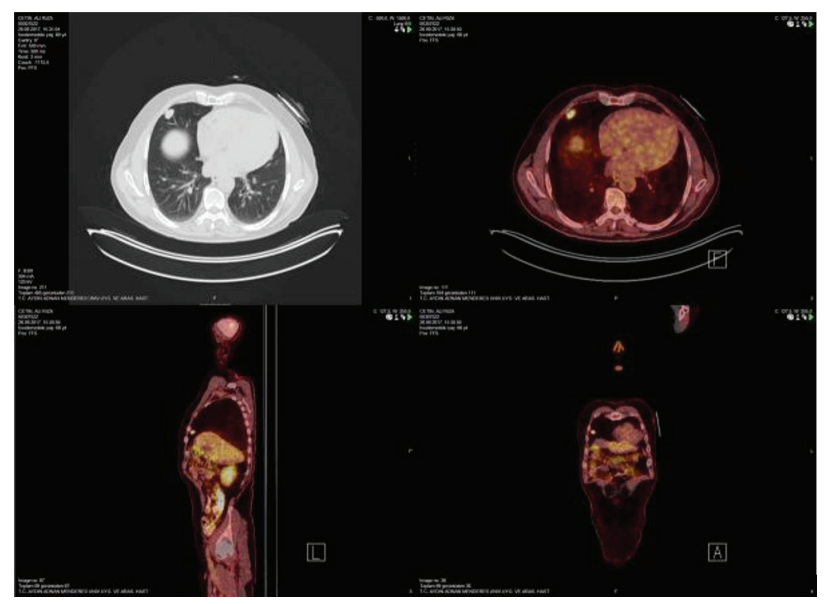

Figure 1. Pulmonary metastasis

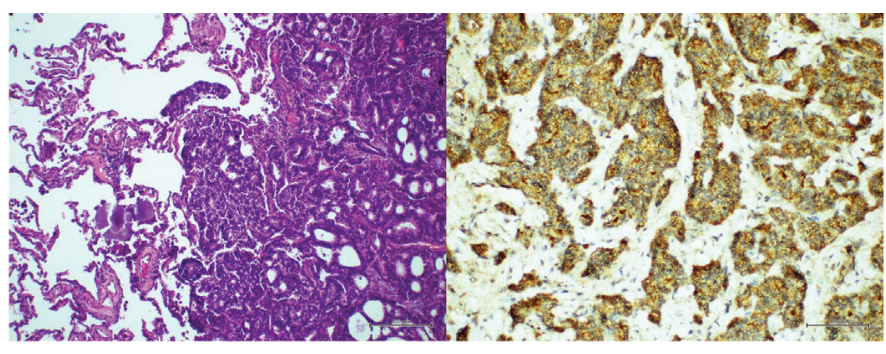

Figure 2. Segmental lobectomy

\section{Discussion}

PCa metastasis mechanism was not fully revealed. Paget (4) proposed the theory of seed and soil, which assumes that metastasis development depends on the interaction between the properties of the metastatic cells (seed) and the characteristics of the target organ microenvironment (soil). The seeds of the PCa metastatic cells are preferably located in the soil of the bone matrix. In addition, the specific target organ attracts cancer cells through the release of chemotactic factors (homing theory) (5). Batson (6) suggested that PCa cells frequently migrate to the skeleton, especially the lower spine, due to a portal-like venous system between the prostate and lower vertebrae. The second most common metastasis site of PCa is the lymph nodes. PCa lymphatic spread always ascends from the pelvis to the retroperitoneum via the common iliac lymph nodes (7). Bubendorf et al. (8) hypothesized that visceral metastases without bone involvement are related to the spread of PCa cells directly through the inferior vena cava, called a cava-type pathway. Recent studies revealed that circulating tumor cells and their count are important in PCa metastasis (9). Tumor cells leading to oligometastatic lesions have not fully achieved their metastatic potential, as the metastatic niche was not fully prepared (10). PCa metastases are seeded not only from the primary tumor but also from other metastatic sites (11). This suggests that curative local treatments are effective in oligometastatic disease.

Immediate or delayed androgen deprivation therapy (ADT) with initial surveillance is preferred for recurrent PCa after curative treatment options (12). Literature has limited high-level evidence comparing survival rates of metastasectomy and ADT (13). In the late 1990s, the hypothesis that metastasis-targeted therapy could increase survival rates was introduced (14). In 2017, Ost et al. (15) published a prospective, randomized multicenter study comparing metastasis-targeted therapy and surveillance in oligometastatic PCa recurrence. Their study started ADT as symptomatic progression, progression to more than three metastases, or local progression of known metastases. They stated that ADT-free survival was longer with metastasistargeted therapy than surveillance alone for oligorecurrent PCa. Metastatectomy and stereotactic body radiotherapy were the most used treatment options for oligometastasis (13). The role of pulmonary metastases resection is still unclear, with few literature results $(16,17)$. A case series by Ciriaco et al. (18) revealed that 1 of 20 patients with oligometastatic $\mathrm{PCa}$, who underwent pulmonary resection, required hormone therapy. The median follow-up period was 23 months and PSA levels were not measurable during the follow-up. Some patients can benefit from this treatment strategy but should be considered only in highly selected patients. Our case preferred metastasistargeted therapy after consulting thoracic surgery for segmental lobectomy, considering surgical complications, ADT-related side effects, and patient conditions.

In conclusion, in oligometastatic PCa, biochemical cure in 2 years follow-up without need for androgen deprivation treatment was evaluated in this case. Visceral metastasis without bone and lymph node involvement in PCa is rare and treatment options are unclear. Metastasectomy in oligometastatic disease has emerged as a treatment option in recent years but is not considered a standard treatment. Literature contributes to define the oligometastatic disease, clarify its nature, and compare treatment options is necessary.

\section{Acknowledgements}

Publication: This study was presented in the $14^{\text {th }}$ International Urooncology Congress on November 6-10 in 2019, Antalya, Turkey.

Contribution: There is not any contributors who may not be listed as authors.

Conflict of Interest: No conflict of interest was declared by the authors.

Financial Disclosure: The authors declared that this study received no financial support. 


\section{Ethics}

Informed Consent: The patient's information was presented as a case report after obtaining patient consent.

Peer-review: Externally peer-reviewed.

\section{Authorship Contributions}

Supervision: H.G., Concept: A.A., Design: A.A., Data Collection or Processing: A.A., M.D., N.Ç., Analysis or Interpretation: A.A., Literature Search: A.A., Writing: A.A.

\section{References}

1. Gandaglia G, Abdollah F, Schiffmann J, et al. Distribution of metastatic sites in patients with prostate cancer: a population-based analysis. Prostate 2014;74:210-216.

2. Gandaglia G, Karakiewicz PI, Briganti A, et al. Impact of the site of metastases on survival in patients with metastatic prostate cancer. Eur Urol 2015;68:325-334.

3. Große Hokamp N, Kobe C, Linzenich E, et al. Solitary PSMA-positive pulmonary metastasis in biochemical relapse of prostate cancer. Clin Nucl Med 2017;42:406-407.

4. Paget S. The distribution of secondary growths in cancer of the breast. 1889. Cancer Metastasis Rev 1989;8:98-101.

5. Jacob K, Webber M, Benayahu D, et al. Osteonectin promotes prostate cancer cell migration and invasion: A possible mechanism for metastasis to bone. Cancer Res 1999;59:4453-4457.

6. Batson OV. The function of the vertebral veins and their role in the spread of metastases. Ann Surg 1940;112:138-149.

7. Briganti A, Suardi N, Capogrosso P, et al. Lymphatic spread of nodal metastases in high-risk prostate cancer: the ascending pathway from the pelvis to the retroperitoneum. Prostate 2012;72:186-192.
8. Bubendorf L, Schopfer A, Wagner $U$, et al. Metastatic patterns of prostate cancer: an autopsy study of 1,589 patients. Hum Pathol 2000;31:578-583.

9. Abalde-Cela S, Piairo P, Diéguez L. The significance of circulating tumour cells in the clinic. Acta Cytol 2019;63:466-478.

10. Celia-Terrassa T, Kang Y. Metastatic niche functions and therapeutic opportunities Nat Cell Biol 2018;20:868-877.

11. Gundem G, Van Loo P, Kremeyer B, et al. The evolutionary history of lethal metastatic prostate cancer. Nature 2015;520:353-357.

12. van den Bergh RC, van Casteren NJ, van den Broeck T, et al. Role of hormonal treatment in prostate cancer patients with nonmetastatic disease recurrence after local curative treatment: a systematic review. Eur Urol 2016;69:802-820.

13. Battaglia A, De Meerleer G, Tosco L, et al. Novel insights into the management of oligometastatic prostate cancer: a comprehensive review. Eur Urol Oncol 2019;2:174-188.

14. Hellman S, Weichselbaum RR: Oligometastases. J Clin Oncol 1995;13:8-10.

15. Ost P, Reynders D, Decaestecker K, et al. Surveillance or metastasisdirected therapy for oligometastatic prostate cancer recurrence: a prospective, randomized, multicenter phase II trial. J Clin Oncol 2018;36:446-453.

16. Gago JP, Câmara G, Dionísio J, et al. Pulmonary metastasis as sole manifestation of relapse in previously treated localised prostate cancer: three exceptional case reports. Ecancermedicalscience 2016;10:645.

17. Wallis CJ, English JC, Goldenberg SL. The role of resection of pulmonary metastases from prostate cancer: a case report and literature review. Can UrolAssoc J 2011;5:E104-E108.

18. Ciriaco P, Briganti A, Bernabei A, et al. Safety and early oncologic outcomes of lung resection in patients with isolated pulmonary recurrent prostate cancer: a single-center experience. Eur Urol 2019;75:871-874. 